\begin{tabular}{|c|c|c|c|c|c|}
\hline 96 ئهi & \multicolumn{5}{|c|}{ Mr. Proctor, on the Shallowness } \\
\hline 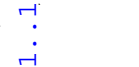 & $\zeta$ Herculis. & & & $\zeta$ Cancri. & \\
\hline 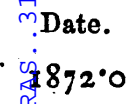 & $\begin{array}{c}\text { Angle of Position. } \\
\text { I8 1 10́ }\end{array}$ & $\begin{array}{c}\text { Distance. } \\
I: 20\end{array}$ & $\begin{array}{l}\text { Date. } \\
1872 \cdot 0\end{array}$ & $\begin{array}{c}\text { Angle of Position. } \\
177^{\circ} 30^{\prime}\end{array}$ & $\begin{array}{c}\text { Distance. } \\
0^{\prime \prime} 6_{2}\end{array}$ \\
\hline $872 \cdot 5$ & 17746 & $1 \cdot 22$ & $1872 \cdot 5$ & 17223 & $0.6 \mathbf{I}$ \\
\hline 望 $873^{\circ} 0$ & 17431 & $I \cdot 24$ & $1873^{\circ} \circ$ & 1678 & 0.60 \\
\hline $\begin{array}{l}1-1 \\
-\pi 873 \cdot 5\end{array}$ & 17121 & $1 \cdot 26$ & $1873 \cdot 5$ & $16 \times 43$ & 0.59 \\
\hline $3874^{\circ} \circ$ & 16812 & $\mathbf{x} \cdot 27$ & $1874^{\circ} \circ$ & $156 \quad 5$ & 0.58 \\
\hline $1874^{\circ} 5$ & 1659 & $1 \cdot 28$ & 1874.5 & 15013 & 0.57 \\
\hline $1875^{\circ} \circ$ & 16212 & $1 \cdot 29$ & $1875^{\circ} 0^{\circ}$ & 1447 & 0.56 \\
\hline
\end{tabular}

\title{
On the Shallowness of the Real Solar Atmosphere.
} By Richard A. Proctor, B.A. (Cambridge).

In my treatise on the Sun I have pointed out at page 192 that the conspicuous nature of the darkening of the disk near the edge is a proof of the shallowness of the superincumbent atmosphere, and not, as is commonly stated, of that atmosphere's being enormously deep. And at p. $295 \mathrm{I}$ indicate reasons for believing that the method by which the prominences and sierra have been studied when the Sun is not eclipsed, is not capable (save under highly exceptional conditions) of exhibiting the existence of the true solar atmosphere, - that atmosphere, to wit, which causes the dark lines in the solar spectrum.

It will be known to all who read this communication that Professor Young, of America, and Mr. Pye, independently recognised the existence of a highly complex atmosphere close by the solar photosphere. The slit of a spectroscope being placed tangentially to the limb, at the place where second contact was to occur, the spectroscopic field at the moment of totality and for several seconds after, was seen to be full of bright lines, "every nonatmospheric line of the solar spectrum showing bright."

The accuracy of this observation has been called in question. It is urged that the method of observing the uneclipsed Sun should be competent to show these bright lines if the supposed atmosphere have a real existence.

Now the competence of the last-mentioned method, so far as its power of obviating the effects of atmospheric illumination is concerned, cannot be questioned. For, indeed, as we know, Mr. Lockyer has, on one occasion, seen multitudes of the Fraunhofer lines reversed in this way. But because he has on all other occasions failed, while neither Zöllner, Respighi, nor Young has been favoured even with a single view of this sort, it is urged that no such atmosphere can exist, or that, at any rate, eclipse observers could have no better opportunity of recognising it with the spectroscope than those who have studied the uneclipsed Sun. I 
Would submit that this inference is erroneous, and that in one important respect observations made during eclipses have a great rad vantage over observations made when the Sun is not eclipsed. it has been overlooked, as I opine, by those who urge the iobjection I am considering, that the image of the solar limb, the telescope, or spectroscopically, is formed by the combination of diffraction-images of the several points of the real limb; and, therefore (independently of irradiation, which, however, should also be taken into account), must needs extend beyond the true outline. We can tell, in fact, how great the extension is, since we know (experimentally) the dimensions of the diffraction-images of luminous points, for given apertures. A telescope which would not separate $\gamma^{2}$ Andromed $\varepsilon$, for instance, would certainly not be capable of showing (when armed with a suitable spectroscope) the bright lines of a solar atmosphere whose height subtend but about the fifth of a second - that is, of an atmosphere 80 or $9 \circ$ miles in height; nor probably would it show these bright lines, even though the atmosphere were three or four times as high.

I think, therefore, that we are not justified in rejecting, or even in regarding as inconclusive, the observation made by Professor Young and Mr. Pye. The inference would clearly be that the sierra cannot in any sense be regarded as the true solar atmosphere. Be it noted, also, that the evidence here considered is altogether independent of that on the strength of which $I$ have been led to assert that in all probability the sierra is not of the nature of a solar envelope at all, but is made up of multitudes of relatively small prominences and of the remains of larger ones. So far as I know, the only circumstance on which the theory that the sierra is an atmospheric envelope was founded, was the supposed smoothness of its outline. This relates to the observations which led to the re-discovery of the sierra in 1868 . But telescopic observations by Airy, Leverrier, Secchi, and many others in $1847,1851,1860$, \&c., had abundantly established the fact that its outline is irregular. Respighi, in 1868 , confirmed this with the spectroscope, and the fact is now generally admitted.

We have, I conceive, no escape from the conclusion that the prominences and sierra consist of glowing vapour which has been flung through the real solar atmosphere, - that atmosphere being highly complex and probably existing, especially near the photosphere, at an enormous pressure. What that medium is in which the prominences and sierra are seen remains to be shown. The idea may be suggested that it is of the same nature as the medium which we call the ether of space. 